\title{
Cáncer de próstata incidental: T1a-T1b. Nuestra experiencia tras observación/intervención radical y revisión de la literatura.
}

\author{
Allué López M, Allepuz Losa C, Borque Fernando A, Serrano Frago P, Gil Martínez P, \\ Gil Sanz M르, Rioja Sanz LA.
}

\author{
Servicio de Urología. Hospital Universitario Miguel Servet. Zaragoza
}

Actas Urol Esp. 2006;30(8):749-753

\section{RESUMEN}

CÁNCER DE PRÓSTATA INCIDENTAL: Tla-T1b. NUESTRA EXPERIENCIA TRAS OBSERVACIÓN/ INTERVENCIÓN RADICAL Y REVISIÓN DE LA LITERATURA.

Objetivos: Analizar el comportamiento del cáncer de próstata Tla y Tlb diagnosticados en nuestro centro.

Material y métodos: Estudio retrospectivo de 40 pacientes en estadio clínico Tla-T1b diagnosticados de adenocarcinoma de próstata en nuestro centro, entre los años 1986 y 1999.

A los 16 pacientes Tla, se les realizó biopsia de reestadificación tras el diagnóstico inicial y control posterior. A los 24 Tlb, se les realizó prostatectomía radical. Todos fueron seguidos mediante tacto rectal y PSA semestral.

Analizamos progresión biológica y/o clínica, tiempo hasta la progresión, mortalidad por causa tumoral y supervivencia.

Resultados: Ninguno de los 16 pacientes con estadio clínico Tla presentó progresión tumoral, con una mediana de seguimiento de 90 meses.

El $12,5 \%$ de los 24 casos $T 1 b$ presentaron progresión tumoral, con una mediana de seguimiento de 70 meses.

La mortalidad cáncer específica fue de un paciente $(4,16 \%)$ que pertenecía al grupo Tlb.

Conclusiones: La observación y seguimiento mediante PSA y tacto rectal del estadio clínico Tla parece ser una buena opción dado el buen pronóstico. De nuestros resultados podría deducirse que los pacientes con estadio clínico Tla y buenos factores pronósticos podrían considerarse con un riesgo de padecer un nuevo cáncer de próstata clínico similar al de la población normal, si bien, son necesarios estudios prospectivos que validen estos resultados.

Los casos Tlb precisan un tratamiento activo y seguimiento más estricto.

Palabras clave: Cáncer de próstata. Cáncer incidental de próstata. Seguimiento.

\section{ABSTRACT \\ INCIDENTAL PROSTATIC CANCER: Tla- T1b. OUR EXPERIENCE AFTER OBSERVATION/RADICAL SURGERY AND LITHERATURE REVIEW}

Objectives: To analyse the progress of Tla and T1b prostate cancer diagnosed in our hospital.

Material and methods: Retrospective study of 40 patients in Tla-T1b clinical stage diagnosed with prostate adenocarcinoma in our hospital, from 1986 to 1999.

A restaging biopsy was performed on the 16 Tla patients after initial diagnosis and control. A radical prostatectomy was performed on the $24 \mathrm{Tlb}$ patients. They were all monitored every six months with rectal exam and PSA.

We analysed biological and/or clinical progression, time to progression, mortality caused by the tumour and survival.

Results: None of the 16 patients with Tla clinical stage presented tumour progression, with a median follow-up of 90 months.

$12,5 \%$ of the $24 \mathrm{T1b}$ cases presented tumour progression, with a median follow-up of 70 months.

Cancer-specific mortality was one patient $(4,16 \%)$ in the Tlb group.

Conclusions: Observation and follow-up with PSA and rectal exam appears to be a good option for Tla clinical stage, given the good prognosis. Our results show that patients with Tla clinical stage and good prognostic factors could be at a similar risk of suffering from a new prostate cancer as the normal population, although prospective studies are required to validate these results.

Tlb cases require active treatment and closer monitoring.

Keywords: Prostatic cancer. Incidental prostatic cancer. Watchful waiting. 
$\mathrm{E}$ cáncer de próstata en estadio Tla y Tlb es un hallazgo histológico incidental tras resección transuretral de próstata o adenomectomía abierta realizadas por patología prostática benigna que no ha sido detectado mediante tacto rectal ni con técnicas de imagen.

Se considera estadio Tla cuando un 5\% o menos del tejido prostático resecado contiene cáncer, y T1b cuando hay tumor en más de un 5\% del tejido. (TNM 2002 ${ }^{1}$ ).

Los factores pronósticos de progresión del cáncer de próstata incidental (Tla-T1b) no están claramente establecidos, por lo que es difícil asegurar cuál es el tratamiento de elección en cada caso.

En el caso del estadio Tla, la observación y seguimiento suele ser el tratamiento de elección en la mayoría de los centros, pero según se describe en la literatura ${ }^{2}$, un bajo porcentaje progresan por lo que se nos podría plantear la posibilidad de aplicar un tratamiento más agresivo en algunos casos seleccionados.

En el estadio Tlb el tratamiento más comúnmente aplicado es de opción curativa (prostatectomía radical, radioterapia,...). Pero a pesar de tratarse de un estadio incipiente y aplicar un tratamiento agresivo, un pequeño porcentaje también progresan.

El objetivo de este estudio es analizar el comportamiento de los casos de cáncer de próstata en estadio clínico Tla y Tlb tratados respectivamente mediante observación o prostatectomía radical en nuestro centro.

\section{MATERIAL Y MÉTODOS}

Se realiza un estudio retrospectivo de 40 pacientes diagnosticados de cáncer de próstata en estadios Tla y Tlb en nuestro centro en el periodo comprendido entre 1986 y 1999 (TNM $2002^{1}$ ).

La edad media de los pacientes estudiados en el momento del diagnóstico fue de 64,43 años (49-71). La mediana de seguimiento de la muestra fue de 79 meses (7-154).

Del total de la muestra, 16 pacientes fueron diagnosticados de cáncer de próstata en estadio T1a, 68,75\% (11 pacientes) mediante resección transuretral de próstata y $31,25 \%$ (5 pacientes) mediante adenomectomía abierta.
Se consideró estadio Tla cuando el 5\% o menos del material resecado contenía tumor. Tras el diagnóstico se realizó biopsia prostática en todos los casos para confirmar el estadio.

El seguimiento de los casos del estadio Tla se realizó mediante PSA y tacto rectal cada 6 meses.

En aquellos casos en los que durante el seguimiento se sospechó progresión tumoral por elevación del PSA ( $>4 \mathrm{ng} / \mathrm{ml}$ o aumento $>1 \mathrm{ng} / \mathrm{ml}$ / año) o alteración del tacto rectal se realizó rebiopsia de próstata y tratamiento posterior según el resultado obtenido.

En los otros 24 pacientes de la muestra se obtuvo un diagnóstico histológico T1b (>5\% del tejido resecado afecto de tumor) tras resección transuretral de próstata (13 pacientes) o adenomectomía abierta (11 casos). A todos ellos se les realizó prostatectomía radical tras el diagnóstico.

El seguimiento posterior de los pacientes con estadio clínico T1b se llevó a cabo mediante tacto rectal y PSA cada 6 meses.

Se consideró progresión el hallazgo de un $\mathrm{PSA}>0,4 \mathrm{ng} / \mathrm{ml}$ tras prostatectomía radical, progresión local o diseminación metastásica.

Analizamos progresión biológica y/o clínica, el tiempo hasta la progresión, la mortalidad cáncer específica y la supervivencia global de los pacientes diagnosticados de cáncer de próstata en estadios clínicos Tla y T1b (Tabla 1).

Tabla 1

\begin{tabular}{lccc}
\hline & & $\begin{array}{c}\text { T1a } \\
\%\end{array}$ & $\begin{array}{c}\text { T1b } \\
\%\end{array}$ \\
\hline Progresión tumoral & Local & 0 & 2 pacientes $(8,3)$ \\
& A distancia & 0 & 1 paciente $(4,16)$ \\
Suma de Gleason & $<7$ & 100 & 83,33 \\
& 7 & 0 & 16,66 \\
Mortalidad cáncer & $>7$ & 0 & 0 \\
específica & & 0 & 1 paciente $(4,16)$ \\
\hline
\end{tabular}

\section{RESULTADOS}

\section{Estadio clínico Tla}

En todos los casos el Gleason clínico fue favorable $(<7)$.

Ninguno de los 16 pacientes con estadio Tla presentó progresión local ni a distancia.

En 3 de los 16 casos $(18,75 \%)$ se precisó la realización de biopsia de próstata durante el 
seguimiento por sospecha de progresión tumoral. El primero de ellos por el hallazgo, a los 3 meses de seguimiento, de un nódulo al tacto rectal. $\mathrm{El}$ segundo caso, por un aumento de consistencia de la glándula prostática al tacto, coincidiendo con un aumento de las cifras de PSA $(>1 \mathrm{ng} / \mathrm{ml} /$ año) a los 3 meses de seguimiento. El último de ellos, ocasionó dudas de progresión tumoral por la elevación de las cifras de PSA al año de seguimiento $>1 \mathrm{ng} / \mathrm{ml} /$ año. En ninguno de los tres casos se confirmó anatomopatológicamente el diagnóstico de cáncer.

\section{Estadio clínico $T 1 b$}

La suma del grado de Gleason clínico de los pacientes en estadio T1b fue $<7$ en el $83,33 \%$ de los casos y de 7 en el 16,66\%.

El estadio patológico tras prostatectomía radical fue en el 20,33\% T0 (no se halló tumor), T2a en el 12,5\%, T2b en el 58,33\%, T3a en el 4,17\% y T3b en el $4,17 \%$.

El 12,5\% (3 pacientes) de los 24 casos diagnosticados inicialmente de estadio clínico $\mathrm{T} 1 \mathrm{~b}$ presentaron progresión tumoral durante el seguimiento. Dos de ellos con progresión biológica a los 42-45,6 meses de seguimiento y con estadios patológicos $\mathrm{T} 2 \mathrm{a}$ y $\mathrm{T} 2 \mathrm{~b}$ respectivamente. $\mathrm{El}$ tercer caso presentó diseminación metastásica a los 7 meses de la intervención (estadio patológico T3b) siendo exitus a los 120 meses tras el diagnóstico de cáncer de próstata (Fig. 1).

La mortalidad cáncer específica en los pacientes con estadio Tla y Tlb fue de $4,16 \%$ ( 1 paciente que pertenecía al estadio Tlb). Ningún paciente del estadio Tla falleció por causa tumoral (Fig. 2).

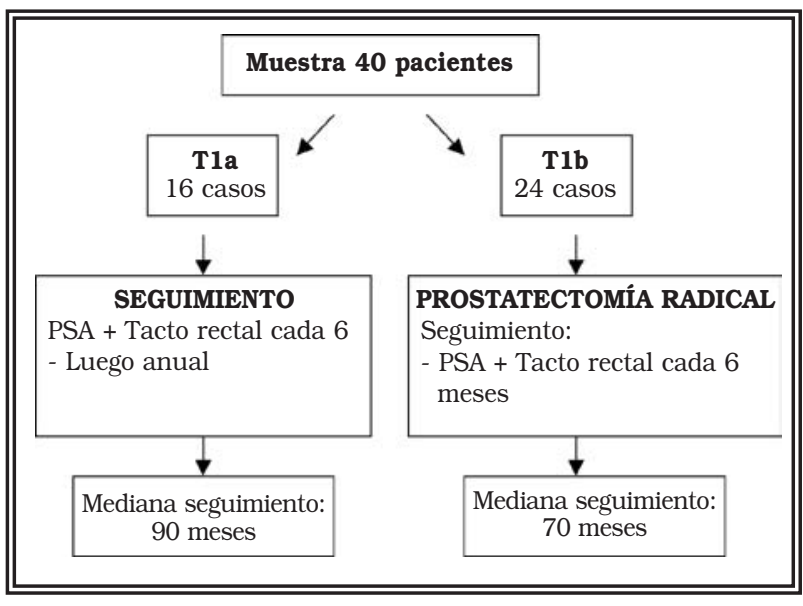

FIGURA 2

\section{DISCUSIŌN}

No está totalmente establecido cuál debe ser la conducta más adecuada ante el hallazgo de un cáncer de próstata incidental (Tla-Tlb).

Según refleja la literatura, en el cáncer de próstata en estadio Tla nos encontramos ante una gran posibilidad de actuaciones tras su diagnóstico: biopsia de próstata o nueva resección transuretral (RTU) para reestadificación del tumor, prostatectomía radical o incluso observación.

Matzkin et al. ${ }^{3}$ en pacientes con supervivencia mayor de 10 años, realizan RTU o biopsia prostática tras el diagnóstico de carcinoma incidental T1a y realizan observación en aquellos con esperanza de vida menor de 10 años. En su serie progresan 16-25\% en 8-10 años.

Chen et al. ${ }^{4}$ en el año 2003, se basan en la suma del grado de Gleason (mayor o menor de 5) de los casos diagnosticados en estadio Tla para optar por un tratamiento radical u observación.

Núñez et al. ${ }^{5}$ en el año 2003, realizan una comparación de la supervivencia y progresión de tumores Tla tratados mediante prostatectomía radical $\mathrm{u}$ observación, sin encontrar diferencias estadísticamente significativas en cuanto a la supervivencia entre pacientes tratados y no tratados.

Kevin et al. ${ }^{6}$ en una revisión de la literatura refieren que la realización de una nueva RTU tras el diagnóstico de cáncer incidental estadio Tla, no debería realizarse de rutina a menos que exista una sospecha de infraestadifica- 
ción del paciente. Varios grupos recomiendan la realización de prostatectomía radical tras el diagnóstico si se trata de pacientes jóvenes. Aquellos pacientes de edad avanzada o comorbilidad importante pueden seguirse mediante observación con PSA y tacto rectal periódicos.

En la última actualización de las Guías Clínicas Europeas ${ }^{2}$ recomiendan observación en aquellos tumores bien o medianamente diferenciados con esperanza de vida $<$ de 10 años. En aquellos con esperanza de vida $>$ de 10 años y con tumor bien o medianamente diferenciado recomiendan reestadificación con ecografía transrectal y biopsia de próstata. Aquellos casos con esperanza de vida $>$ de 10 años \pm tumor mal diferenciado se deben tratar mediante prostatectomía radical o radioterapia (RT).

En nuestro grupo de pacientes hemos realizado reestadiaje mediante biopsia de próstata inmediatamente tras el diagnóstico de cáncer de próstata en estadio Tla, siendo la observación con tacto rectal y PSA el seguimiento escogido.

El porcentaje de progresión en estadio Tla tras observación, varía según las series publicadas. Así las Guías Clínicas Europeas ${ }^{2}$ reflejan un $5 \%$ de progresión a 5 años y hasta $50 \%$ a $10-13$ años. Otros autores rebelan hasta un $37 \%$ de progresión a los 10 años ${ }^{7}$.

Ninguno de los pacientes Tla de nuestro estudio ha progresado, por lo que consideramos que la observación podría ser un manejo suficiente en estos casos. Además, los Tla sin malos factores pronósticos probablemente podrían ser considerados con un riesgo de padecer un nuevo cáncer de próstata similar al de la población normal, pudiendo asemejarse su seguimiento a la de ésta. La mortalidad cáncer específica en nuestra muestra es nula con una mediana de seguimiento de 90 meses.

Esta disminución de las cifras de progresión halladas podrían estar ocasionadas por una mejor estadificación actual, debido a que en nuestro centro se realiza biopsia de próstata de reestadificación a todos los pacientes y que además en la actualidad se toman un mayor número de cilindros prostáticos; quizá de este modo estudiamos mejor y descartamos aquellos tumores más agresivos, disminuyendo así el porcentaje de progresión. Hay que tener en cuenta que los porcenta- jes de progresión reflejados en las Guías Clínicas y en otros artículos ${ }^{2,7}$ corresponden a estudios comprendidos entre 1992 y 1998.

En cuanto al estadio T1b, el riesgo de progresión reflejado en la literatura, es mucho mayor que para $\mathrm{Tla}$, progresando en un porcentaje no despreciable a pesar de un tratamiento agresivo como prostatectomía radical o radioterapia tras el diagnóstico. Así Timbal et al. ${ }^{8}$ encuentran una progresión del $29 \%$ a los 17 meses de media tras la realización de próstatectomía radical. Manseck et al. ${ }^{9}$ hallan un $19 \%$ de progresión a los 10 años tras cirugía radical, con una mortalidad cáncer específica del $12,5 \%$.

En nuestra serie, $12,5 \%$ de los casos progresan tras una mediana de seguimiento de 70 meses. La mortalidad cáncer específica es de $4 \%$.

Según todos estos datos podemos concluir que el estadio T1b presenta un \% de progresión importante, por lo que justifica un tratamiento más activo (cirugía radical, radioterapia,...) y un seguimiento más estricto.

\section{CONCLUSIONES}

En conclusión y debido a los resultados que hemos obtenido, creemos que en los cánceres de próstata Tla la observación y seguimiento mediante PSA y tacto rectal parece una buena opción. Aquellos pacientes con ausencia de factores pronósticos negativos podrían ser considerados con el mismo riesgo de padecer un nuevo cáncer de próstata que la población normal, por lo que sería posible realizar un seguimiento igual a ésta.

Los estadios clínicos $\mathrm{T} 1 \mathrm{~b}$ requieren un tratamiento activo (prostatectomía radical, radioterapia...) y un seguimiento estricto.

\section{REFERENCIAS}

1. Greene FL, Page DL, Fleming ID, Fritz A, Balch CM, Haller DG, et al. AJCC Cancer Staging Handbook: from the AJCC Cancer Staging Manual. 6th ed. New York: Springer; 2002.

2. EAU (European Association of Urology) Guidelines on Prostate Cancer 2005. Disponible en: URL:http://www.uroweb.nl/files/uploaded_files/2005ProstateCancer.pdf

3. Matzkin H, Patel JP, Altwein JE, Soloway MS. Stage T1A carcinoma of prostate. Urology 1994;43(1):11-21.

4. Chen WM, Yang CR, Ou YC, et al. Clinical outcome of patients with stage Tla prostate cancer. Division of Urology. J Chin Med Assoc. 2003;66(4):236-240. 
5. Núñez López AV, Ojea Calvo A, Domínguez Freire F, Alonso Rodrigo AE, Rodríguez Iglesias B, Benavente Delgado J, et al. Clinical and biological progresion of incidental prostatic cancer (Stage T1a). Actas Urol Esp. 2003;27(5):345-349.

6. Kevin R, Andrew A, Sanjaya Kumar. Expectant management of stage A-1 (Tla). Prostate Cancer utilizing Serum PSA levels: a preliminary report. Journal of Surgical oncology 1999;70:49-53.

7. Bostwick DG. The Pathology of incidental carcinoma. Department of Pathology, Mayo Clinic, Rochester, MN 55905, USA. Cancer Surv. 1995;23:7-18.

8. Tombal B, Visccher L, Cosyns F, Lorge R, Opsomer R, Wese $\mathrm{F}$, et al. Assessing the risk of unsuspected prostate canser in patients with bening prostatic hypertrophy: a 13-year

\section{COMENTARIO EDITORIAL}

Los autores abordan un interesante estudio sobre el carcinoma prostático incidental (Tla y T1b), estadio este con profundas implicaciones científicas y clínicas.

Aceptando las limitaciones propias de todo trabajo retrospectivo y con un circunscrito número de casos, diversas consideraciones útiles y de interés pueden extraerse del mismo. Así, ningún paciente en estadio Tla falleció por cáncer de próstata. Dado que el grado Gleason fue en todos los casos menor de 7 , como oportunamente consignan los autores, la observación coincide con el comportamiento registrado en la bibliografía para este estadio. Lamentablemente, la ausencia de casos con Gleason alto impide conocer cual hubiera sido su posible tendencia evolutiva.

En el caso de los $\mathrm{T} 1 \mathrm{~b}$, resulta interesante señalar la pobre correlación existente entre la estadificación inicial y la definitiva, la cual llega a incluir un $20 \%$ de TO. Respecto a la posible mala evolución de estos casos, es muy oportuna la diferenciación señalada por los autores según retrospective study of the incidence and natural history of T1a.T1b prostate cancers. BJU International 1999;84:10151020.

9. Manseck A, Wirth M, Theiss M, Frohmuller H. Radical prostatectomy in patients with incidental prostate carcinoma. Urol Int. 1994;53(4):196-208.

Dra. M. Allué López

e-mail: martaallue@yahoo.com

(Trabajo recibido el 18 de abril de 2006)

que el grado Gleason sea mayor o menor de 7. Por eso mismo sería de gran interés constatar si hubo relación entre dicho grado y progresión de la enfermedad. Esta relación, de confirmarse, sumada al alto porcentaje de pTO, podría matizar la necesidad de tratamiento radical sistemático para el estadio Tlb.

No debe olvidarse que un aspecto relevante en este tipo de estudios es el estado vital de los enfermos al final del seguimiento. En este artículo se detallan muy correctamente los pacientes fallecidos durante el seguimiento, pero no se distingue entre pacientes perdidos, muertos por otra causa y retirados vivos al cierre del estudio, datos estos que se podrían investigar en ulteriores revisiones del tema.

Mientras no se disponga de los datos definitivos de los diversos trabajos prospectivos en marcha, artículos de este tipo siempre con un riguroso planteamiento metodológico, pueden ser de gran utilidad para el manejo de los enfermos con cáncer prostático incidental.

Fdo. Alfredo Ipiéns Aznar 